\title{
Anabases
}

ANABASES Traditions et réceptions de l'Antiquité

$3 \mid 2006$

Varia

\section{L'ethnographie romaine et ses primitifs : les paradoxes de la « préhistoire » au présent}

\section{Pierre Cordier}

\section{(2) OpenEdition}

1 Journals

Édition électronique

URL : http://journals.openedition.org/anabases/2716

DOI : 10.4000/anabases.2716

ISSN : 2256-9421

Éditeur

E.R.A.S.M.E.

\section{Édition imprimée}

Date de publication : 1 mars 2006

Pagination : 173-193

ISSN : 1774-4296

\section{Référence électronique}

Pierre Cordier, «L'ethnographie romaine et ses primitifs : les paradoxes de la « préhistoire » au présent ", Anabases [En ligne], 3 | 2006, mis en ligne le 01 janvier 2012, consulté le 20 octobre 2019. URL: http://journals.openedition.org/anabases/2716 ; DOI : 10.4000/anabases.2716 


\section{L'ethnographie romaine et ses primitifs : les paradoxes de la " préhistoire " au présent}

PierRe Cordier

LA TRADITION PHILOSOPHIQUE grecque fournit la trame de toute réflexion romaine sur l'état originel de l'Homme ; les débats des philosophes sur l'éternité du monde, sur les cycles ou les tendances de l'histoire humaine, sur l'animalité de l'homme originel, sa qualité morale, les atouts ou les handicaps dont la Nature l'a doté en mère attentive ou en cruelle marâtre ressurgissent bien sûr dans les écrits des philosophes latins (de Lucrèce à Saint Augustin, en passant par Cicéron et le Songe de Scipion), mais aussi dans la littérature ethnographique, dont ils fournissent l'arrière-plan théorique ${ }^{1}$. Dans le problème qui nous occupe - la préhistoire des Anciens, la façon dont les Anciens rendent compte de l'anthropogenèse et/ou de l'apparition de l'état social -, il faut commencer par souligner le caractère fondamental de l'histoire des théories, de leur circulation et de leur évolution ; cette approche doxographique génère des arbres généalogiques et retrace le cheminement des traditions intellectuelles. Pour autant, l'identification des antécédents, des modèles et des contre-modèles, des inspirations et des polémiques n'épuise pas la question, en particulier parce que, tout à la poursuite de son objet (l'archétype), elle néglige par définition la fonction des altérations ou des infléchissements apportées à telle ou telle doctrine. Pour illustrer ce point, après l'illustration qu'en a donnée José Kany-Turpin dans le présent volume, je me contenterai d'un seul exemple, que j'emprunterai au livre $\mathrm{V}$ du De rerum natura de Lucrèce :

1 Pour un aperçu de ces traditions, cf. A. O. LOVEJOY, J. BOAS (éd.), Primitivism and related ideas in Antiquity (Contributions to the history of primitivism, 1), Baltimore, The John Hopkins Press, 1935 (prolegomena, p. 1-22), réimpr. 1997 et, dans le présent volume, M.-L. Desclos, "Représentations platoniciennes du temps d'avant" et J. KaNYTuRPIN, "Notre passé antérieur prophétisé ?". 
Et l'enfant ? semblable au matelot que les flots furieux ont rejeté sur le rivage, il gît, tout nu, par terre, incapable de parler, dépourvu de tout ce qui aide à vivre, dès l'heure où, le projetant sur les rives que baigne la lumière, la nature l'arrache du ventre de sa mère : de ses vagissements plaintifs il remplit l'espace, comme il est juste à qui la vie réserve encore tant de maux à traverser ! Au contraire, on voit croître sans peine les animaux domestiques, gros et petits, et les bêtes sauvages : ils n'ont besoin ni de hochets, ni des mots caressants que chuchote la voix d'une tendre nourrice; ils ne sont pas en quête de vêtements qui changent avec les époques de l'année ; enfin ils n'ont point besoin ni d'armes ni de hautes murailles pour défendre leurs biens, puisque pour parer à tous leurs besoins, la terre et la nature inventive enfantent d'elles-mêmes toutes sortes d'abondantes ressources ${ }^{2}$.

Les animaux, selon Lucrèce, n'ont nul besoin d'artéfacts (nec... opus est) pour atteindre leur plein développement. Bien dotés dès la naissance de toutes les facultés physiques et de toutes les ressources alimentaires nécessaires à leur survie par la Nature, qui se comporte avec eux en bonne mère, ils poussent sans effort, contrainte ni difficulté - spontanément, comme feraient des végétaux - Mais avec l'homme, la nature s'est faite marâtre. Dépendant d'autrui dès la seconde de sa naissance (indigus omnil uitali auxilio), le bébé n'est qu'une boule de chair et de souffrance, livré tout entier à la détresse. Le besoin lui tient lieu de dispositions naturelles. À peine venu au monde, l'enfant se manifeste par un cri. Il y a là comme l'expression prémonitoire d'une angoisse existentielle constitutive de l'espèce. Ce tableau des premiers instants de la vie humaine est bien sûr un lieu commun, héritage d'une longue tradition philosophique ${ }^{3}$. Mais en latin, le topos prend une couleur particulière. L'image du bébé gisant nu, à même le sol, appelle irrésistiblement la réminiscence antiquaire d'une coutume qui prenait place au sein des rites de naissance romain. Avant sa prise en charge au sein de la domus et son intégration à l'entourage familial, le tout-petit devait être déposé nu à même le sol. Le père était censé, si c'était un garçon, le prendre dans ses bras (c'est le geste de tollere infantem) ou donner l'ordre de la nourrir (ali jubere) si c'était une fille. Ces opérations sanctionnaient la reconnaissance du nouveau venu et ouvraient la séquence des rites de naissance par lesquels commence le long travail de socialisation de

2 Lucrèce V, 222-234, tr. J. KANY-TuRPIN (éd.), Lucrèce. De la nature. De rerum natura, Paris, Flammarion, éd. revue, 1998.

3

Sur le thème de la natura nouerca et de l'homme comme nudus infans, cf. E. POEHLMANN, "Der Mensch das Mängelwesen ?”, Archiv für Kulturgeschischte 52 (1970), p. 297-312 ; J.-P. Néraudau, Etre enfant à Rome, Paris, Belles Lettres, 1984, p. 70-74 ; B. RocheTTE, “Nudus... infans... : à propos de Lucrèce, V, 222-227”, Les Etudes Classiques 60 (1992), p. 63-73 et, dernièrement, P. CORDIER, Nudités romaines. Un problème d'histoire et d'anthropologie (Études anciennes, série latine, 63), Paris, Belles Lettres, 2005, p. 22-50. Pour le thème chez Platon en particulier, cf. F. Vonessen, "Die Mutter als Stiefmutter. Zur Mythologie eines Märchenmotivs”, Symbolon 1 (1972), p. 113-137. 
l'enfant romain. En l'absence de ces gestes rituels et de la volonté expresse, manifestée par le père, d'accepter l'enfant, ce dernier pouvait être abandonné au fumier du carrefour (c'est l'expositio) ${ }^{4}$.

Ainsi l'argumentaire philosophique de Lucrèce se dédouble-t-il : l'exemple du bébé établit que, par nature, contrairement à l'animal, l'homme non seulement met des années à devenir ce qu'il est, mais s'avère même incapable d'y parvenir tout seul. La nudité de l'être humain à la naissance, figurant la misère de la condition humaine, est en même temps le principe positif de sa marche vers une dignité qui peut l'élever audessus du règne animal. Bien que sa nudité originelle signifie à l'Homme son dénuement et sa faiblesse devant ses appétences, elle met en place, dès l'origine, un terrain favorable à la sociogenèse et au progrès humain par un " pacte de la nature " (foedus naturae) 5 .

À l'interprétation de cet épisode dans les termes de la tradition philosophique, il faut superposer un autre mode de lecture : dans un contexte culturel romain, cette séquence oppose l'abandon au lien social. La nudité, condition brute du corps et degré zéro de la socialisation ${ }^{6}$, contraste avec les soins à caractère rituel, indissolublement liés à la définition du groupe gentilice et, à travers lui, à une conception de l'identité romaine à la fois comme communauté normée et comme dynamique d'acculturation (cultus). En d'autres termes, le texte de Lucrèce est susceptible de se dédoubler : reflet de traditions philosophiques grecques, il peut en même temps être lu comme la justification philosophique, ou plutôt la naturalisation de modes de socialisation distinctifs de la romanité 7 . Cet exemple illustre une stratégie identitaire caractéristique des élites romaines, capables de penser en Grecs tout en gardant la pose du Romain. La philosophie épicurienne fournit de la sorte, en se développant au sein de la latinité, l'armature d'une anthropologie romaine.

Ce bébé romain, résumé vivant et paradigme incarné de la misère et de la grandeur humaines, et cette entrée en matière un peu trop longue prétendent introduire la

4 Cf. T. KöVes-Zulauf, Römische Geburtsriten (Zetemata, 87), Munich, Beck, 1990, p. 1-91 ; F. DUPONT, "Le lait du père romain”, in P. MOREAU (éd.), Corpsromains, Grenoble, Jérôme Millon, 2002, p. 115-138 ; Y. THOMAS, "À Rome, pères citoyens et cités des pères", in A. Burguière, C. Klapisch-Zuber, M. Segalen, F. Zonabend (éd.), Histoire de la famille, 1, Mondes lointains, Paris, A. Colin, 2001, p. 195-229, notamment p. 257.

5 Cf. G. DrOZ-Vincent, "Les foedera naturae chez Lucrèce", in C. LÉvy (éd.), Le concept de nature à Rome. La physique, Paris, Presses de l'École normale supérieure (Études de littérature ancienne, 6), 1996, p. 191-211, notamment p. 196-197 ; cf., plus haut, l'article de J. KANY-TuRPIN, "Notre passé antérieur prophétisé ?".

6 Sur la nudité comme figure de la désocialisation, cf. CORDIER, Nudités romaines, p. $120-125$ et $148-154$.

7 Sur la naturalisation des catégories sociales, cf. S. MACDONALD, "The Socialisation of the Natural and the Naturalisation of the Social”, in S. ARDENER (éd.), Defining Females. The Nature of Women in Society, Oxford, Berg, 1993,p. 287-305. 
question centrale de mon exposé, celle de la façon dont la culture romaine se pose le problème de l'homme primitif, de la sociogenèse ${ }^{8}$ et du passé antérieur de l'état social.

Parler d'ethnographie romaine ${ }^{9}$ ne va pas de soi : même dans des ouvrages encore récents et bien documentés, elle semble en effet inexistante, écrasée entre une Antiquité grecque fertile en théories et en textes fondateurs et un Moyen Âge assez clairement chrétien pour trancher nettement avec les demi-teintes du monde antique tardif 10. Cette évanescence de l'ethnographie romaine tient, pour beaucoup, au pouvoir de fascination d'une évidence (les savoirs romains sont ancrés dans des traditions grecques) et à la vigueur légitime de la tradition doxographique. Mais l'un des enjeux majeurs de la question qui nous occupe est de tenter d'isoler un faisceau de représentations distinctif de la romanité dans un tissu de traditions partout imprégné d'hellénisme - il ne s'agit en aucun cas de sacrifier à l'illusion d'un fait social romain pur et sans mélange, qu'il suffirait d'extraire par décantation, mais au contraire de chercher à comprendre comment un discours spécifiquement romain s'élabore en incorporant des éléments

8 Qu'il me soit permis de désigner ici par le terme de sociogenèse le développement du comportement social non pas chez l'individu, comme le veut le sens ordinaire du mot, mais dans l'espèce en général.

9 Le terme d'“ethnographie" peut prêter au malentendu. Assurément, l'ethnographie des Anciens rapporte une expérience de l'Altérité (cf. F. HARTOG, Le miroir d'Hérodote, Paris, Gallimard, 1980, rééd. 2001), emprunte la forme d'une représentation par l'écriture qui transforme le regard en langage (cf. F. LisSaRRague, "Graphein. Scrivere e disegnare", Graphica 3 [1987], p. 10-19). Elle se concentre généralement sur la perception des phénomènes sociaux, mais aussi d'une forme de globalité - elle prend toujours en compte, par exemple, l'environnement écologique dans la définition de son objet, l'étranger. Mais, parce qu'elle est étrangère à l'idée de relativité des cultures, donnée fondamentale des sciences sociales contemporaines (cf. D. CUCHE, La notion de culture dans les sciences sociales, Paris, La Découverte, 1996, p. 3-67), elle ne s'accompagne d'aucun retour réflexif sur le point de vue de l'observateur, d'aucun effort - ou d'aucune illusion - d'effacement de soi ; la description elle-même est paramétrée selon des références culturelles qui s'imposent au réel extérieur et le modèlent, et dont un régime constant d'intertextualité est le symptôme le plus visible, comme on le constatera dans les exemples développés au cours du présent article.

10 Cf. par exemple le chapitre "L'Antiquité chrétienne et le Moyen Âge chrétien. La difficulté du regard sur l'Autre", dans M. KILANI, Introduction à l'anthropologie, Lausanne, Payot, 1992, p. 195-200. 
empruntés qu'il réinterprète : comment, en somme, Rome fait du romain avec du grec, quand il s'agit de donner corps à l'idée de civilisation 11 .

Bien des angles d'approche étaient possibles. J'ai volontairement choisi le discours en apparence le plus empirique et le moins systématique dans son usage des références philosophiques, celui de l'ethnographie. L'ethnographie n'échappe pas aux contraintes des autres genres littéraires antiques : la saturation par l'autorité des Anciens, la prédilection pour les antithèses et les figures exemplaires, l'imprégnation du récit par des enjeux moraux ${ }^{12}$. La description des étrangetés contemporaines n'échappe pas à ces tendances, mais, plus que les exempla traditionnels, elle est confrontée à la diversité du réel : aux marges de l'aire culturelle gréco-romaine, les communautés de chasseurscueilleurs ou les sociétés proto-politiques différaient sensiblement selon leur environnement ${ }^{13}$.

Dans la littérature ethnographique romaine, je m'attacherai aux sociétés contemporaines expressément identifiées par les observateurs ou les compilateurs comme étrangères à l'organisation politique, qu'elles consistent en communautés paléolithiques de chasseurs-cueilleurs ou que leur organisation les situe au seuil de la cité, comme les Germains de César. La contemporanéité de ces sociétés explique les modalités de leur présentation. Elles apparaissent volontiers dans des excursus au sein de récits historiques ou de traités ; leur image montre moins, en transparence, cette épaisseur de mémoire, cette enfilade de signaux d'intertextualité qui enracine la tradition ethnographique de

11 Il n'est pas question ici de ressusciter le débat franco-allemand opposant " culture " et " civilisation ", cf. N. Elias, La civilisation des mours, Paris, Calmann-Lévy, 1973 (tr. fr. par P. Kamnitzer de la première partie de Über den Prozess der Zivilisation, 1939, p. 11-73). Par le terme de "civilisation ", j'entends seulement rendre compte des prétentions universalistes que développe la culture romaine, cf. P. VEYNE, "Humanitas: les Romains et les autres", in A. GIARDINA (éd.), L'homme romain, Paris, Seuil (tr. de L'Uomo romano, Rome I Bari, Laterza 1989), 1992, p. 437-478 ; W. SPEYER, "Reale und ideale Oikumene in der griechischen und römischen Antike", Wiener Studien 114 (2001), p. 449-462. Par culture, je désigne seulement l'ensemble de connaissances, de comportements et de façons de faire distinctifs d'une organisation sociale - et reconnus comme tels par ses acteurs -.

12 F. LasSERre, s. v. "Ethnographie", Lexikon der Alten Welt, Zürich, Stuttgart, Artemis Verlag, 1965, p. 886-890 ; D. TimPE, "Ethnologische Begriffsbildung in der Antike", in H. BECK, Germanenprobleme in heutiger Sicht (Ergänzungsbände zum Reallexikon der Germanischen Altertumskunde, 1), Berlin, De Gruyter, 1986, p. 22-40 : P. JANNI, s. v. "Ethnographie", in H. Sonnabend, Mensch und Landschaft in der Antike. Lexikon der historischen Geographie, Stuttgart, J.B. Metzler, 1999, p. 126-133.

13 Par "proto-politique ", je désigne ici les organisations " semi-étatiques " d'A. TESTARD, Éléments de classification des sociétés, Paris, Errance, 2005, p. 106-113. Le terme de " protopolitique " veut faire ressortir la perspective romaine sur l'évolution des sociétés, cf. infra. 
l'Antiquité, malgré ses lectures modernes, parmi les productions de la paideia antique et dans ses façons de faire distinctives 14 .

C'est désormais un lieu commun que de souligner le double mouvement que révèle la description ethnographique chez l'observateur. Il est fasciné par une irréductible altérité ; en même temps, il expérimente, de façon délibérée ou inconsciente, l'évidence de ses propres catégories culturelles, leur puissance d'attraction et leur faculté de $s^{\prime}$ imposer au réel ${ }^{15}$. La saisie de l'altérité des peuples paléolithiques ou proto-politiques n'est pas le seul enjeu du discours ethnographique. La description étonnée, méprisante ou emphatique de ces peuples contemporains, limitrophes de l'imperium Romanum, voire enclavés en son sein, signale à la fois l'expérience de l'altérité et celle de l'hétérogénéité du temps vécu. Expérience de l'espace et du dépaysement, le discours ethnographique propose aussi l'expérience d'un Temps mosaïqué et hétérogène, en juxtaposant, aux yeux de l'observateur et de son lectorat, des époques différentes dans un paysage simultané : la découverte des espaces "primitifs " est perçue comme un bond en arrière dans le temps 16 .

En historicisant la différence, en y intégrant le temps (leur présent, c'est notre passé), l'ethnographie romaine, non moins que celle des colonisateurs européens du XIXe et de la première moitié du XXe siècle, met en jeu une conception de la culture romaine (cultus) comme dynamique d'acculturation et légitime son universalité. Loin que l'hétérogénéité du temps historique aboutisse à relativiser l'Histoire, l'existence, dans le présent, de mondes " préhistoriques " est un signal indiquant le sens de sa marche. C'est pourquoi, pour évoquer les groupes humains sous le regard de l'ethnographie romaine, il n'est pas illégitime de recourir au lexique d'une ethnographie contemporaine désormais datée, pour les qualifier de "primitifs ». Ces anachronismes volontaires peuvent s'avérer fructueux, à condition de s'arrêter sur le mode d'emploi et d'assigner à leur emploi analogique des limites et des précautions. Ils ont l'avantage d'éviter l'emploi du mot de "barbare ", qui estompe la sensation de l'écart temporel dans la perception de l'Autre.

14 Il s'agit, pour beaucoup, d'une illusion entretenue par la conservation sélective des auteurs de l'Antiquité, même compte tenu d'une tendance générale du discours ethnographique à produire des stéréotypes : cf. K. HASTRUP, "Anthropology and the Exaggeration of Culture", Ethnos 50 (1985), p. 313-324.

15 Cf. Cuche, La notion de culture, s. v. "ethnocentrisme ", p. 21.

16 Bien loin de souscrire à cette hiérarchie temporelle, je veux seulement souligner son caractère profondément subjectif - c'est-à-dire liée à l'expérience que l'observateur fait de l'évidence et de la centralité de sa propre culture. Il faut souscrire aux remarques de Johannes Fabian sur les illusions et les arrière-pensées confortables que recouvre, dans le discours ethnographique, le sentiment, chez le voyageur découvrant l'altérité, d'un saut dans le temps et d'une régression vers une époque primitive : Time and the Other, New York, Columbia University Press, 1983, p. 17-18 et passim. 
Le terme français de "primitif ", à partir du XVIe siècle, prend un sens temporel, et n'est employé à la fin du XVIII siècle pour qualifier ce qui est proche de l'état de nature, ce qui reste mal dégrossi et proche des premiers âges, et enfin les sociétés non occidentales ${ }^{17}$. Dans son emploi substantivé, le mot est promu au XIXe siècle par l'ethnologie évolutionniste : il évoque un registre culturel resté proche de l'état originaire de l'humanité ${ }^{18}$. Le mot tend à disparaître du vocabulaire anthropologique, en raison des connotations attachées à cette origine. Il reste employé néanmoins, en particulier chez Lévi-Strauss, pour désigner désormais une antériorité non historique, mais logique à la

17 Cf. A. REY (dir.), Dictionnaire historique de la langue française, II, Paris, Dictionnaires Le Robert, 1992, p. 1632 et ID., Dictionnaire culturel en langue française, III, Paris, Dictionnaires le Robert, 2005, p. 2070-2071. Pour une histoire de la catégorie de " primitif " en anthropologie, cf. A. KUPER, The Invention of Primitive Society, Londres, Routledge, 1988, éd. révisée 2005. Pour une série d'approches sectorielles de la catégorie de "primitif " dans les lettres, l'art et les sciences humaines, cf. F. McInTOSHVARJABÉDIAN (éd.), Discours sur le primitif (UL 3 Travaux et recherches), Villeneuve d'Ascq, Université Charles-de-Gaule-Lille III, 2002. Le terme allemand de " Naturvölker ", communément utilisé en allemand pour désigner ce que les anthropologues français appellent les "peuples primitifs " et les anglo-saxons " primitive people ", connaît une évolution plus précoce. Forgé à la fin du XVIII siècle par J.G. HERDER (cf. A. LÖCHTE, Johann Gottfried Herder. Kulturtheorie und Humanitätsidee der Ideen, Humanitätsbriefe und Adrastea (Epistemata Philosophiae, 540), Stuttgart, Königshausen \& Neumann, 2005, p. 100-127), dans le contexte d'un débat philosophique sur la nature et la culture, pour s'opposer aux "Kulturvölker », il perd dès la seconde moitié du XIXe siècle les connotations péjoratives dont il pouvait être affecté pour désigner une culture à part entière. Le glissement sémantique semble imputable à la somme de T. WhaITZ, Anthropologie der Naturvölker, Leipzig, F. Fleischer, 1859-1871, œuvre rédigée entre 1844 et 1864, cf. C. MAIGNÉ, "Anthropologie der Naturvölker de Theodor Waitz (1859) : une anthropologie sans races”, in C. TraUTMAnN-Waller (dir.), Quand Berlin pensait les peuples, Anthropologie, ethnologie et psychologie (1850-1890), Paris, CNRS Éditions, 2004, p. 197-210. Mais jusque dans la seconde moitié du XXe siècle, le terme reste lourd de hiérarchies implicites, comme en témoigne la mise au point introductive de L. Frobenius, Kulturgeschichte Afrikas. Prolegomena zu einer historischen Gestaltlehre. Zürich, Phaidon Verlag, 1954, p. 10-17.

18 Les racines de cette idée sont anciennes : il suffit de citer le jésuite Joseph François LAFITAU (1681-1746) et ses Mours des Sauvages amériquains comparées aux mours des premiers temps (Paris, 1724), étudié par M. DE CERTEAU, "Histoire et anthropologie chez Lafitau", in C. BlanCKaert (éd.), Naissance de l'ethnologie ? Anthropologie et missions en Amérique, XVIe-XVIII siècle, Paris, Cerf, 1985, p. 62-89. Cf. aussi A.C. TAYLOR, s. v. "primitif ", in P. Bonte et M. IZARD, Dictionnaire de l'ethnologie et de l'anthropologie, Paris, Presses Universitaires de France, 1991, p. 600-601. 
manière de l'" état de nature » dans le Discours sur l'origine de l'inégalité de Rousseau ${ }^{19}$. La catégorie évolutionniste de " primitif ", en dépit de son caractère anachronique, n'est pas foncièrement inadéquate pour nommer des formes sociales dans lesquelles les récits romains reconnaissent des stades antérieurs de leur propre organisation sociale. Peu importe que l'impression d'antériorité résulte d'un raisonnement inductif et fasse appel plus ou moins consciemment à des schémas préconstruits du passage à l'état de société - historiques, philosophiques ou religieux - : cette désignation organise bel et bien les formes sociales en une séquence chronologique et en une hiérarchie de valeurs. Dire que les Romains avaient leurs primitifs revient à constater qu'au contact de ces derniers, leur propre culture leur apparaissait comme un processus évolutif : la civilisation. L'emploi du terme de "sauvage 20 " appelle moins de scrupules, si l'on se souvient qu'il prétend traduire le latin ferus, fréquemment employé pour qualifier des groupes sociaux dont l'organisation est illisible ou foncièrement étrangère et, par certains de leurs usages, proches de l'animalité 21 .

Les écrits des Romains antérieurs au IIe siècle ne nous sont guère parvenus. Pour l'essentiel, la littérature romaine est postérieure aux guerres puniques, à la dilatation de l'imperium Romanum à travers le monde méditerranéen, voire à la guerre sociale et à la brutale expansion du corps civique qui s'ensuivit. Parmi les bouleversements de toute nature qui accompagnèrent ces changements d'échelle et trahissent la crise de croissance traversée par le monde romain, des phénomènes apparemment contradictoires se distinguent : les élites du nouveau monde romain, enrichies par les profits de la conquête, ornées d'une culture valorisante, l'hellénisme, et aspirées par l'Vrbs se dotent progressivement de nouvelles règles de comportement : l'urbanitas, qui érige en idéal les mœurs policées, les savoirs distingués, tous les raffinements de la Ville ${ }^{22}$.

19 C'est en ce sens que C. LÉVI-STRAUSS cherche à réhabiliter le terme, dans son Anthropologie structurale, Paris, Plon, 1958, p. 114 : "Le terme de "primitif" semble définitivement à l'abri des confusions impliquées par son sens étymologique et entretenues par un évolutionnisme périmé. Un peuple primitif n’est pas un peuple arriéré ou attardé. » Pour une critique de la tendance, chez Lévi-Strauss, à objectiver cet état théorique, cf. J. Copans, "Lévi-Strauss face à Rousseau ou la censure du politique", in J.-L. AMSELLE (éd.), Le sauvage à la mode, Paris, Le Sycomore, 1979, p. $27-94$.

20 Cf. Rey (dir.), Dictionnaire historique de la langue française, II, p. 1885-1886 ; ID., Dictionnaire culturel en langue française, IV, 2005, p. 581-587. Pour une histoire récente du stéréotype dans la perspective de la psychologie sociale, G. JAHODA, Images of Savages : Ancient roots of modern prejudice in Western culture, Londres, Routledge, 1999.

21 C'est ainsi que Cicéron qualifie la sodalité des luperques (fera sodalitas) et Tacite la condition des Fennes (ferae sortis), cf. infra.

22 E. Valette-Cagnac, "Plus attique que la langue des Athéniens. Le grec imaginaire des Romains”, in F. Dupont, E. Valette-Cagnac (éd.), Façons de parler grec à Rome, Paris, Belin, 2005, p. 37-80, notamment p. 55-63. 
Simultanément, au sein de ces mêmes élites, se fait jour l'angoisse d'une déperdition identitaire, l'idée que la civilisation est une entropie, agissant comme une maladie sur un corps social qu'elle dépouille peu à peu des caractères natifs auxquels il doit sa position actuelle : dans les eaux tièdes et confortables de la culture, la langueur, l'amnésie, la dissolution guettent le Romain ${ }^{23}$. La génération de Cicéron offre bien des exemples de ce tiraillement entre les standards de vie du présent et l'idéal identitaire d'une tradition antique devenue opaque et fuyante; parmi ses contemporains, le personnage de l'antiquaire Varron est une figure emblématique de cette aventure ambiguë 24.

\section{Comment peut-on être germain ?}

La question du "primitivisme " romain naît dans ce contexte culturel, à la fois comme un symptôme de la crise et comme un effort de résolution. Un passage fameux de la Guerre des Gaules de César, qui présente les mœurs des Germains en les opposant à celle des Gaulois, en fournit un exemple significatif ${ }^{25}$.

Les mœurs des Germains sont très différentes de cette coutume. En effet, ils n'ont ni druides pour présider au culte des dieux (qui rebus dininis praesint) ni pratique des sacrifices. Au nombre des dieux, ils ne comptent que ceux qu'ils voient et dont l'aide et l'assistance sont patents, le Soleil, Vulcain, la Lune ; quant aux autres, ils n'en ont même pas entendu parler. Toute leur vie tient en chasses et en pratiques militaires; dès leur petite enfance, ils ont la pratique de la peine et de l'endurcissement. Plus longtemps ils sont restés impubères, plus ils sont honorés par leur entourage : on devient ainsi, selon les uns, plus grand, plus fort, plus nerveux selon les autres. De fait, connaître la femme avant l'âge de vingt ans passe chez eux pour l'une des pires turpitudes. La chose pourtant n'est aucunement dissimulée, car hommes et femmes se baignent indistinctement dans les rivières, et d'ailleurs ils ne se servent pour se couvrir que de peaux ou de courts rénons, la plus grande partie du corps à nu.

Ils n'ont pas la pratique de l'agriculture, et leur alimentation consiste surtout en lait, fromage et viande. Personne ne possède en propre une étendue fixe de terrain, un domaine à soi, mais les magistrats et les chefs (magistratus ac principes) attribuent chaque fois pour une année aux clans et aux parentèles (gentibus cognationibusque hominum) vivant ensemble l'étendue et l'emplacement de terrain qui leur semble bon et, l'année suivante,

23 Sur la culture comme facteur pathogène, cf. D. GOUREVITCH, Le mal d'être femme, Paris, Belles Lettres, 1984, p. 39-43.

24 J'emprunte intentionnellement l'expression d' " aventure ambiguë " au beau roman de Cheikh Hamidou Kane, L'aventure ambiguë, Paris, Julliard, 1961. Sur Varron, cf. C. Moatti, La raison de Rome. Naissance de l'espritcritique à la fin de la République, Paris, Seuil, 1997, p. 143-145.

César, $B G \mathrm{VI}, 21-23$. Je traduis. 
ils les forcent d'aller ailleurs. Ils donnent maintes raisons de cet usage : crainte d'être poussés par la continuité de la coutume à troquer la pratique de la guerre contre l'agriculture ; de pratiquer l'accaparement, de laisser les forts chasser les faibles de leurs possessions (possessionibus) ; de bâtir trop soigneusement pour se protéger du froid et de la chaleur ; de voir naître l'amour de l'argent, qui engendre factions et des dissensions ; désir enfin de tenir la plèbe (plebem) par la sérénité, chacun se voyant, pour la fortune, l'égal des plus puissants.

Pour les cités (ciuitatibus), il n'est pas de plus grand honneur que d'avoir autour de soi la plus vaste étendue possible de désert et de confins dépeuplés. À leurs yeux, le signe distinctif de leur valeur, c'est que leurs voisins, chassés de leurs champs, émigrent, et que personne n'ose demeurer près d'eux ; ils voient là en même temps une mesure de sécurité, n'ayant plus à craindre d'une invasion subite. Quand une cité repousse une guerre qu'on lui fait ou la porte chez autrui, on choisit des magistrats pour conduire cette guerre, avec le droit de vie et de mort (magistratus qui ei bello praesint et uitae necisque habeant potestatem deliguntur). Mais en temps de paix, il n'y a pas de magistrat en commun, mais les chefs de régions et de cantons (principes regionum atque pagorum) rendent la justice et apaisent les litiges chacun chez soi. Les larcins n'ont rien d'infâme, quand ils sont commis en dehors des frontières de chaque cité ; ils proclament que c'est un moyen d'exercer les jeunes gens et de réfréner chez eux la paresse. Et lorsqu'un chef, dans une assemblée, se propose pour commandant, invitant qui veut suivre à se déclarer, ceux qui approuvent et la proposition et l'homme se dressent, s'engagent à l'aider, et ils sont honorés à l'unisson par toute l'assistance ; mais ceux d'entre eux qui ne suivent pas, on les compte pour des déserteurs et des traîtres, et ils perdent leur crédit (fides) en toute chose. Porter atteinte à un hôte, c'est à leurs yeux contraire à l'ordre du monde : les gens qui, pour une raison quelconque, viennent chez eux, ils les protègent de l'injustice, les tiennent pour sacrés ; pour ces gens, toutes les maisons sont ouvertes et tous les repas partagés.

La nature de l'information de César reste problématique. Le tableau est probablement hybride, composé d'allusions au début du livre XXX des Histoires de Poseidonios d'Apamée, pour s'en tenir à des référents identifiables, mais aussi de données relevées par César ou ses lieutenants et relevant de l'autopsie ${ }^{26}$. Sans relever de la fantaisie pure,

26 Sur le livre XXX de Poseidonios, cf. M. LAFFRANQUE, Poseidonios d'Apamée (essai de mise au point), Paris, PUF, 1964, p. 120, et n. 49 ad loc. Sur la conception de la société, chez Poseidonios, p. 494-508 ; cf. Sénèque, Ad Lucilium 90, 5. Cf. aussi J. J. Tierney, "The Celtic Ethnography of Poseidonius", Proc. Royal Irish Academy 60 (1960), p. 189-275 ; ID. et D. NASH, "Reconsidering Poseidonius. Celtic Ethnography : some considerations", Britannia 7 (1976), p. 111-126 ; J. WEBSTER, "Ethnographic Barbarity : colonial discourse and Celtic warrior societies", in J. WEBSTER et N. COOPER (éd.), Roman Imperialism : Post-Colonial Perspectives (Leicester archaeological monographs 3), Leicester, 1996, p. 111-124. Le texte de César appelle également des rapprochements avec l'évocation des Spartiates chez Xénophon, République des Lacédémoniens, II, 3-7. 
les détails ethnographiques montrent des échos de la conception posidonienne de l'âge d'or, telle du moins que Sénèque l'a transmise : le pouvoir est aux mains d'hommes prudents, les responsables de la communauté assumant leur fonction de tutelle sans en tirer d'avantages matériels. Mais la référence érudite à un état proche de l'humanité primitive est ambiguë. Elle constitue à la fois une qualité - celle d'une nature pas encore corrompue par la civilisation - et un défaut - celle d'une communauté inachevée, instable et, à moins de changer, condamnée à rester asociale. Cette ambivalence du primitivisme traduit la difficulté de concevoir le moment du changement.

Le portrait des Germains, dans son ensemble, a la raideur d'un système de stéréotypes et les présente comme une nation demeurée en deçà du politique : s'ils montrent une organisation sociale susceptible d'analogies avec les structures de la cité (ciuitas), il ne s'agit que d'une cité embryonnaire, encore inachevée : les magistrats (magistratus), comme la propriété, sont mouvants, instables, circonstanciels. Le pouvoir des commandants militaires n'est qu'une ébauche d'imperium, sans régularité annuelle ni composante civile, tandis que le principe de la vie civile reste la communauté de résidence. Les Germains ont de la religion une pratique intuitive, embryonnaire, caractérisée par une structuration incomplète qui la laisse toujours au seuil de la cinitas : ils s'en tiennent à vénérer les forces visibles de la nature, ignorant l'institution des sacerdoces. Leur haute moralité de primitifs, le cas qu'il font d'une virginité préservée le plus longtemps possible, leur permettent d'accepter avec innocence qu'hommes et femmes se baignent nus ensemble, mais cette mixité même trahit le stade primitif, encore indifférencié, d'une vie communautaire insoucieuse de la division des sexes. De même, l'absence de propriété personnelle et la rotation des biens immobiliers entre les Germains, réputé garantir l'égalité entre les membres de la communauté, ne livre qu'un produit chiche et rudimentaire. Tout agriculteurs qu'ils sont, ils ont un régime alimentaire de nomades : du lait, du fromage et de la viande, mais céréales et légumes manquent à l'inventaire (on se souvient que les Romains se reconnaissaient dans le sobriquet de "mangeurs de bouillie [puls] ", et qu’à Dyrrhachium, pendant la guerre civile qui opposa César à Pompée, le régime de disette des césariens, mangeurs de végétaux sauvages et de lait, fut utilisé pour démoraliser les Pompéiens) 27. La nature particulière de leurs institutions révèle leur sauvagerie intrinsèque : ils ne sont capables de se doter de magistrats centraux que pour la guerre, et ignorent les institutions civiles communes à tous. Seule la violence sait les souder ensemble, mais cette cohésion reste temporaire. Cet inachèvement politique les rend incapables de coexister avec qui que ce soit : ils ne savent vivre quelque part qu'après avoir fait tout à l'entour le vide autour d'eux, n'ont de respect que

27 Le mangeur de bouillie : Plaut., Most. 828 (pultiphagus), Poen. 54 (pultiphagonides). Sur Dyrrhachium : César, $B C$ III, 47-48. La ration des soldats était normalement composée de blé (frumentum) ou, en cas de manque, de viande (César, $B G \mathrm{VII}, 17,3 ; B C \mathrm{I}, 48,6$ et 52,4$)$. Le régime végétarien et lacté des césariens était un signe de leur acharnement, mais aussi d'une forme de barbarie contractée au cours des années de campagne en Gaule. 
pour la propriété garantie par leur propre coutume et tiennent pour un sain exercice le vol exercé aux dépens d'autrui. Étrangers, dans leur naïve entièreté, à toute forme de tromperie, ils attachent à la parole donnée une valeur définitive, ignorant toute forme de casuistique. Les vertus de leur sauvagerie sont des qualités, qui appellent des noms romains (uirtus, fides, frugalitas, pudicitia), à l'instar de leurs frustes institutions (imperium, religio), mais ces qualités ne sont pas parvenues à maturité et, telles quelles, trahissent une société inaboutie. Proches de la condition primitive de l'homme, les Germains de César servent à la fois de repoussoir aux vertus nationales romaines, que la civilisation a portées à leur perfection, et de repère permettant de mesurer l'écart entre ces vertus idéales et la corruption des mœurs du temps.

\section{Gens sans terre : le peuple du no man's land}

Dans un ouvrage récent, Trevor Murphy a mis en évidence le dénominateur commun des développements ethnographiques que présente l'Histoire naturelle de Pline : tous présentent des peuplades que distingue une carence dominante et, pour ainsi dire, caractérisées par une forme singulière d'inachèvement ${ }^{28}$. C'est le cas des Chauques, un peuple de Germanie septentrionale, qui occupaient une zone entre la Weser et l'Elbe ${ }^{29}$. L'intérêt de leur description chez Pline est d'autant plus grand qu'il s'agit, aux dires du naturaliste même, d'une autopsie ${ }^{30}$. Pour autant, il faut se garder d'un enthousiasme excessif : aux dires de Pline le Jeune, son fils adoptif, l'érudit avait composé des Bella Germaniae en vingt livres ${ }^{31}$. Il était donc au fait, autant qu'on pouvait l'être, des traditions historiques, géographiques et ethnographiques relatives à la Germanie, et son développement n'a rien d'une confrontation brutale avec un monde insoupçonné.

Il conviendrait de parler à la suite aussi des arbres à glands, qui ont fourni le premier aliment des mortels et ont nourri leur condition misérable et sauvage ( $q u<a>e$ primae

T. Murphy, Pliny the Elder's Natural History. The Empire in the Encyclopedia, Oxford, Oxford University Press, 2004, p. 165 : "Savages embody various kinds of lack - lack of names, lack of dreams, lack of institutional virtues like shame, continuity, and stability, or a simple and final lack of humanity ."

29 G. Neumann, R. Wenskus et R. SCHMid, s. v. "Chauken", Reallexikon der Germanischen Altertumskunde, zweite Auflage, IV, Berlin, De Gruyter, 1981 p. 393-413.

Plin., HNXVI, 1. Cf. K. Sallmann, "Reserved for Eternal Punishment: The Elder Pliny's View of Free Germania (HN 16.1-6)”, American Journal of Philology 108 (1987), p. 108128 ; Murphy, Pliny the Elder's Natural History, p. 168-174. Sur les Chauques, cf. aussi Tacite, Annales XI, 19 et Ptolémée II, 11, 7, 2. Pline avait découvert les Chauques pendant l'expédition de Corbulon en 47, cf. E. Norden, Die Germanische Urgeschichte in Tacitus, Leipzig, Teubner, 1920, p. 207-311 ; MurPHY, Pliny the Elder's Natural History, p. 166169.

Pline le Jeune, Lettres III, 5. 
victum mortalium aluerunt nutrices inopis ac ferae sortis), si je ne devais d'abord noter l'étonnement que j'ai éprouvé en voyant comment pouvaient vivre des hommes sans aucun arbre, sans aucun arbuste. Nous avons dit qu'en Orient du moins plusieurs nations riveraines de l'Océan sont réduites à cette nécessité. Mais dans le Nord aussi, nous avons $\mathrm{vu}$ (sunt vero et in septentrione visae nobis) les Chauques, qu'on appelle grands et petits. Là, par un immense mouvement, deux fois en vingt-quatre heures, l'océan se répand et s'étale à l'infini, recouvrant le théâtre de l'éternelle question posée par la nature, région qu'on ne sait attribuer à la terre ou à la mer. Là, nation misérable, ils occupent des tertres élevés ou des tribunes dressées de leurs mains d'après leur expérience de la plus haute marée ; ils y installent leurs cases et, semblables à des navigateurs quand les eaux recouvrent les alentours, à des naufragés quand elles se sont retirées, ils poursuivent autour des huttes des poissons qui s'enfuient avec la mer. Ils ne peuvent avoir de bétail ni se nourrir de lait comme leurs voisins, ni même se battre contre les bêtes sauvages, éloignés qu'ils sont de tout arbrisseau. Avec des laîches et des joncs des marécages, ils tressent des cordes pour faire des filets à poissons; ils ramassent de la boue à la main, la font sécher aux vents plus qu'au soleil, et exposent au feu de cette terre leurs aliments et leurs entrailles raidies par le froid nordique. Leur seule boisson est de l'eau de pluie conservée dans des trous à l'entrée de leur demeure. Et ces nations, si elles sont aujourd'hui vaincues par le peuple romain, crient à l'esclavage ! En vérité, souvent la fortune épargne ceux qu'elle veut punir.

Lévocation des Chauques est appelée par la description de leur milieu naturel, dépourvu d'arbres et tiraillé entre la terre et la mer ${ }^{32}$. Ce paysage stérile n'est pas anodin : il plante le décor d'une communauté dont le principe fédérateur et l'industrie quotidienne est la gestion d'une pénurie de chaque instant. La vie misérable de ces Germains du littoral est une véritable malédiction. Il faut souligner dans quels termes Pline évoque l'absence de végétaux ( $q u<a>e$ primae victum mortalium aluerunt nutrices inopis ac ferae sortis). Il y a là, à l'évidence, un rappel de traditions relatives aux origines de l'humanité, quand l'homme tirait sa subsistance de la seule cueillette, la nature lui prodiguant le nécessaire 33 . Le territoire désolé des Chauques ne fournit aucun matériau susceptible de les armer pour la chasse. Nul gibier dans ce désert, sinon les hommes eux-mêmes, livrés sans défense aux attaques des fauves. La guerre de l'homme contre les animaux, stade primitif de la maîtrise par l'homme de son milieu écologique selon Lucrèce, n'est même pas envisageable. La vie des Chauques, en somme, donne à voir l'œuvre, amplifiée de manière hyperbolique, d'une marâtre nature qui les prive de tout moyen de développer les savoir-faire et les modes de vie propres à l'humanité. Bien loin

32 C'est la dimicatio naturae, cf. Murphy, Pliny the Elder's Natural History, p. 169 et ID., n. 8.

33 Dans le présent volume, M.-L. Desclos, "Représentations platoniciennes du temps d'avant" et J. KANY-TURPIN, "Notre passé antérieur prophétisé ?". 
d'admirer l'adaptation des Germains à cet environnement hostile, Pline présente leur entêtement à occuper des lieux aussi invivables comme le signe d'une inaptitude au développement.

Le naturaliste laisse entendre que cette incapacité n'est pas seulement l'effet de la pénurie de tous les biens nécessaires à la mise en place d'une structure sociale digne de ce nom ; elle résulte aussi du choix fait par le peuple du littoral. Il persévère en effet dans son mode de vie absurde et stérile et n'envisage à aucun prix d'en changer, quand bien même on lui apporterait la civilisation à domicile. L'excursus s'achève précisément sur l'arrivée des Romains, en qui les misérables laissés pour compte de la culture devraient acclamer leurs libérateurs ; loin de là, ces primitifs refusent la promesse du progrès et crient à la servitude. Le développement de Pline sur les Chauques, bien qu'il se donne pour une autopsie, laisse transparaître, du début à la fin, des modèles philosophiques qui constituent son infrastructure et influencent sa réception ${ }^{34}$. Parce qu' elle répond à l'entêtement scandaleux que les primitifs nordiques mettent à conserver leur société " froide ", la conquête romaine est légitime. Elle réalise en effet un développement qui aurait dû s'accomplir, crée les conditions nécessaires pour que s'achève chez les Chauques un processus de socialisation - pour ne pas dire d'hominisation - demeuré inachevé. La conquête de la Germanie par Rome prolonge et accomplit de la sorte l'œuvre de la Nature, qui veut que l'homme aille vers la culture au lieu de se complaire aux confins de l'animalité ${ }^{35}$. Quand, contre des primitifs invétérés dans leur misère culturelle, les hostilités ont pour visée une telle entreprise de civilisation, elles ne sauraient lancer qu'une guerre juste 36 .

\section{Sous les poux, la sagesse barbare}

La littérature ethnographique impériale développe des tendances analogues à la schématisation morale des caractères qu'elle relève chez les peuples ignorant l'organisation politique. Dans une parenthèse de sa Germanie, Tacite évoque, parmi les peuplades du grand Nord, les Fennes, ancêtres des Finnois, qui se distinguent de son environnement germanique par leur sauvagerie 37 .

34 Les données matérielles et la hiérarchie culturelle dont Pline fait état ne s'accordent pas avec les trouvailles archéologiques : durant les premiers siècles ap. J.-C., le littoral des Chauques livre un matériel plus riche que celui de l'arrière-pays, cf. SALLMANN, "Reserved for Eternal Punishment: The Elder”, p. 116.

P. VeYNe, "Humanitas : les Romains et les autres" ; P. Gros, "Le barbare humanisé ou les limites de l' humanitas", in C. Auvray-Assayas (éd.), Images romaines, Paris, Éditions rue d'Ulm, 1998, p. 143-160. 
Tac., Germ. XLVI Les Vénèthes ont pris beaucoup de leurs mœurs, car tout ce qui s'élève de monts et de forêts entre Peucins et Fennes, est rempli de leurs courses et de leurs brigandages ; pourtant on les compte plutôt parmi les Germains parce qu'ils plantent leurs maisons, portent des boucliers, se plaisent aux manœuvres d'une infanterie légère, bien différents en cela des Sarmates qui passent leur vie en chariot et à cheval. Chez les Fennes, étonnante sauvagerie, hideuse misère (mira feritas, foeda paupertas), saleté de tous, torpeur des grands; pas d'armes, pas de chevaux, pas de pénates ; pour nourriture, l'herbe, pour vêtement, des peaux, et comme lit, la terre; leurs seuls espoirs sont dans leurs flèches qu'ils appointent, par manque de fer (inopia ferri), avec des os. La même chasse nourrit également les hommes et les femmes; car elles les suivent partout et réclament leur part dans la quête. Et les nourrissons n'ont d'autre abri contre les bêtes et contre les pluies qu'un couvert de branches entrelacées; c'est aussi la retraite des jeunes gens, l'asile des vieillards. Mais ils trouvent cette condition plus heureuse que d'ahaner sur des champs, peiner à des maisons, soupeser dans la crainte et dans l'espoir leurs biens avec ceux des autres. En sûreté du côté des hommes, en sûreté du côté des dieux, ils sont parvenus à l'état le moins accessible aux mortels : ils n'ont même plus de vœux à former.

Le portrait des Fennes, véritable catalogue de carences, est le négatif de la civilisation ; ils ignorent les techniques de la guerre, ils n'ont pas de domicile fixe, pas de vêtements tissés, pas de mobilier de confort, ils ne savent rien de la métallurgie et se contentent d'abris provisoires de feuillage ; il n'y a chez eux ni espace, ni activité propre aux femmes, ni séparation des générations : tous se mélangent dans des espaces indistincts, et cette indétermination est le signe d'un défaut de socialisation. Le monde des Fennes, ainsi réduit à une enfilade d'absences et de carences, est proche du degré zéro de l'humanité. Mais la phrase qui clôt la description les transforme en une allégorie philosophique : dépouillés de tout, n'ayant rien à perdre, les Fennes incarnent la sécurité absolue. Une expression proverbiale, attestée dès Plaute, mais aussi, sous la forme d'une sentence philosophique, chez Sénèque, fait de la nudité la condition la plus enviable, la garantie d'un esprit affranchi de tout souci, le signe d'un détachement moral absolu : la securitas 38 . Tacite va jusqu’à renchérir sur la pauvreté des Fennes : ils sont au-delà de la pauvreté, dans un état chronique de carence tel qu'ils se font les mendiants les uns des autres : leur frugalité philosophique dépasse de loin la condition

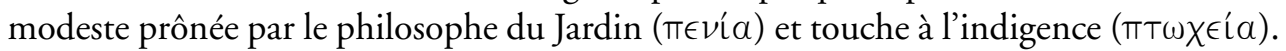
Le dénuement des Fennes est l'envers de l'opulence romaine et de ses passions créées par la civilisation, l'image d'une condition idéale que rien ne saurait troubler. Chez

38 Plaut., Asinaria 92-93 Maxumas nugas agis : / nudo detrahere vestimenta me iubes: "Calembredaines! Tu me demandes de déshabiller un homme tout nu "; cf. aussi Sen., Ad Lucilium 31, 10 Parem autem te deo pecunia non faciet : deus nihil habet. Praetexta non faciet : deus nudus est: " Mais l'argent ne fera pas de toi l'égal du Dieu : le dieu n'a rien. La toge prétexte ne le fera pas : le dieu est nu. "Cf. CORDIER, Nudités romaines, p. 138147. 
Pline, la dévalorisation de la sauvagerie était motivée par le contexte de la confrontation entre l'observateur et les Chauques : une entreprise de conquête romaine et son refus par les Germains. La description de Tacite s'inscrit dans un tout autre environnement : situés en dehors de l'imperium et destinés à y rester, les Fennes n'interferent pas avec le monde du cultus, restant, pour ainsi dire, anhistoriques. Dès lors, ils se prêtent à l'idéalisation et aux variations moralisantes sur les vertus de l'humanité primitive.

Dans ces deux exemples, l'ambiguïté du primitivisme ethnographique romain apparaît nettement : dans leur rudesse, les mœurs des primitifs sont inquiétantes, voire vaguement répugnantes. Pourtant, elles mettent en évidence les tares ou les formes de déperdition qui accompagnent le processus de civilisation. Dans son ambiguïté, le primitivisme se contente de renvoyer dos à dos la civilisation par défaut des primitifs et la civilisation par excès des contemporains trop raffinés. Il opère en confrontant deux stéréotypes, un brouillon d'homme et un modèle déjà altéré par la civilisation. Toute perspective historique est absente de cette confrontation ou, plutôt, la seule dimension historique tient dans la conscience d'un écart, d'un temps de latence entre l'excès de civilisation et l'équilibre idéal entre civilisation et sauvagerie qu'ont connu un jour les Romains. Mais le point d'équilibre fuit en un point indéfinissable de l'histoire, et il n'est jamais évoqué que d'une façon indirecte, comme un moment critique abstrait, pour rendre compte de la tension entre le temps d'avant la culture et le temps d'après. La synkrisis ethnographique semble ainsi se contenter d'enchaîner sur une ligne théorique les valeurs approchées de l'humanité. On aboutit à un paradoxe : en dépit de son apparence empirique, au plus près de la diversité des phénomènes, elle semble converger avec les modèles de l'anthropogenèse élaborés au sein de systèmes philosophiques.

Cette inclination philosophique de l'ethnographie romaine tient à la difficulté de concevoir le moment critique de la civilisation, s'agissant de soi-même. Un Romain ne saurait se représenter Rome que comme cité. Mais il suffit que Rome soit une cité pour que l'équilibre soit rompu et que la romanité ait déjà basculé du côté de la civilisation. C'est cette pétition de principe qu'énonce Cicéron dans un texte fameux du De re publica, cherchant à écarter des traditions relatives à la fondation de Rome le grief de l'affabulation naïve. Rome n'est pas devenue une cité, mais elle l'a été dès son origine, comme sortie tout armée de la tête de Romulus ${ }^{39}$ :

L'époque de Romulus, elle, se place il y a moins de six cents ans, et nous savons bien qu'alors l'écriture et l'instruction étaient depuis longtemps connues et que les égarements anciens de l'humanité primitive avaient disparu.

Significativement, le point de repère choisi pour évaluer la position de l'Vrbs sur l'échelle de la civilisation n'est pas ici la sauvagerie des peuples préhistoriques, mais un autre univers contemporain, la culture hellénique (Cicéron place la fondation de Rome, 
selon Timée, en l'an deux de la septième olympiade, c'est-à-dire en 884 av. J.-C.). Le raisonnement est osé : puisque les Grecs, à cette époque, ont atteint un haut degré de civilisation, alors Rome est a principio une cité dont les traditions sont fiables. Soit Rome est une cité grecque, soit le génie de Romulus a su, d'emblée, tirer la quintessence de la civilisation contemporaine la plus avancée. Dans tout les cas, le moment critique de la civilisation fuit en amont, régressant dans le temps.

\section{Qui craint les grands méchants loups?}

Rome serait-elle une communauté sans préhistoire ? Non, mais une communauté dont la pensée de la préhistoire ou, si l'on veut, des formes prépolitiques, sont devenues inaccessibles ou insupportables pour un Romain. Le même Cicéron, jamais à court de contradictions, propose ailleurs une évocation des Romains préhistoriques, à travers la figure des Luperques, les jeunes membres de la sodalité qui, durant les fêtes de février, couraient nus, les hanches ceintes d'une peau de bouc fraîchement sacrifié, du Lupercal, vers le Forum Boarium, puis l'Ara Maxima, l'autel de Consus, les Curiae ueteres, et le sanctuaire des Lares avant de revenir à leur point de départ ${ }^{40}$.

Fera quaedam sodalitas et plane pastoricia atque agrestis germanorum Lupercorum, quorum coitio illa siluestris ante est instituta quam humanitas atque leges. Confrérie vraiment grossière, franchement pastorale et rustique, que celle de ces frères Luperques, dont l'association fut formée autrefois dans les bois avant la civilisation et la législation.

Les Luperques sont aux yeux de Cicéron des résurgences incongrues d'un univers précivique et d'un monde de sauvagerie heureusement lointain. Leur accoutrement de peaux évoque la silhouette de pasteurs, figures de nomades étrangers à l'agriculture et au monde des territoires définis ${ }^{41}$. Mais les Lupercales ne montrent pas une Arcadie de salon. Les luperques vont au-delà de la sauvagerie policée des bergers : leurs peaux sont brutes et crues ${ }^{42}$. Le principe de la communauté qu'ils forment est un lien familial entre germains (germani). Ce lien de sang les constitue comme un groupe fermé, imperméable aux échanges sociaux ; ils constituent ainsi une société embryonnaire, un clan analogue à celle que forment les bêtes féroces dans les forêts, leur communauté

40 Cic., Cael. 26 Clark.

41 Sur l'étrangeté des pasteurs à l'époque de Cicéron, cf. A. DEman, "Bergers transhumants et mouvements de résistance en Italie depuis les Gracques jusqu’à César”, T. YUGE, M. DOI (éd.), Forms of Control and Subordination in Antiquity. Proceedings of the International Symposium for Studies on the Ancient World, Tokyo, January 1986, Leyde, Brill, 1988, p. 209225.

42 Cf. CoRdier, Nudités romaines, p. 154 sur les vêtements de peau des esclaves bergers. Ils étaient considérés par le droit romain comme un vêtement, c'est-à-dire une marque de socialisation concédée par le maître (Digeste, 34, 2, 23, 2 Ulp. libr. XLIV ad Sabinum). 
empruntant la forme la moins évoluée de socialisation ${ }^{43}$. La compagnie sauvage (fera... sodalitas) des luperques est une harde, le degré zéro de la société à qui l'humanité et les lois n'ont pas encore donné la forme harmonieuse d'une ciuitas.

Le commentaire de Cicéron sur cette embarrassante sodalité véhicule discrètement une étymologie, ou au moins une analogie, qui donne les luperques pour des loups, figures emblématiques de la sauvagerie (feritas), qui s'opposent à l'homme comme des jumeaux anomiques 44 . À la différence d'autres modèles de romanité, comme celui que développaient les imperatores dans leurs harangues aux troupes, en associant la ferocia ou la feritas à la discipline militaire (disciplina), le modèle cicéronien n'avait que faire de la sauvagerie. C'est pourquoi la fête des Lupercales dégoûtait vaguement Cicéron, parce que son rituel mettait en scène la résurgence d'un état prépolitique incompatible avec l'idée que l'Arpinate se fait de Rome, cité de l'humanitas. Ce n'était pas une simple affectation : ces rituels poussaient à la transgression et tendaient, même bien circonscrits au contexte de la fête, à brouiller les catégories sociales. Quelques décennies plus tard, Auguste devait mettre un terme au scandale des Lupercales, en remplaçant les jouvenceaux ceints de peau de bouc sanguinolentes par des chevaliers romains vêtus de pagnes au plissé compliqué et probablement frottés d'huile ${ }^{45}$. Signe des temps, la disposition savante des plis et l'onction, usage cosmétique d'inspiration grecque, affichaient clairement, tout comme la dignité équestre des sodales, le signal de l'état de culture (cultus), voire de sa forme la plus raffinée et la plus distinguée, l'urbanitas. Pour Auguste, qui empruntait à Cicéron une grande partie de son argumentaire, la préhistoire de Rome n'était pas " bonne à penser ».

\section{Vitruve et la carte du Temps}

Nous avons cru constater que l'ethnographie romaine convergeait avec les modèles philosophiques de l'anthropogenèse, apparemment, en réduisant à une simple probabilité abstraite la transition historique de l'état primitif à l'état de culture. Mais il n'en va pas toujours ainsi. Un exemple étonnant en fait foi : les pages que Vitruve consacre, en matière d'éloge de l'architecture, à l'apparition de l'art de bâtir, première œuvre et exercice de l'intelligence humaine, à l'origine de tous les arts.

43 Cf. dans le présent volume, M.-L. Desclos, "Représentations platoniciennes du temps d'avant".

44 Sur les rapports étymologiques divers proposés par les sources anciennes et les auteurs contemporains entre lupus et lupercus/lupercal, cf. G. DUMÉZIL, La religion romaine

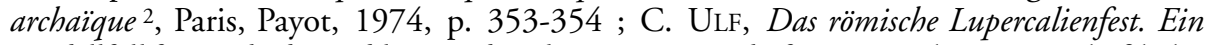
Modellfall für Methodenprobleme in der Altertumswissenschaft, Darmstadt, Wissenschaftliche Buchgesellschaft, 1982, p. 11-24. 
Vitr., De arch. II, 1, tr. L. Callebat, (CUF, 1990) :

Les hommes primitifs naissaient, comme les animaux sauvages, dans les forêts, dans les grottes, dans les bois et n'avaient d'autre nourriture pour vivre que les aliments offerts par la nature. Il arriva quelque part que des arbres, en masse serrée, battus à coups redoublés par les vents des tempêtes, frottant leurs branches les unes aux autres, firent jaillir le feu : terrifiés par cette flamme violente, ceux qui étaient près de l'endroit s'enfuirent. Puis, quand le phénomène s'apaisa, ils s'approchèrent et, constatant le grand bien-être que donnait à leur corps la tiédeur du feu, ils l'entretinrent en y ajoutant du bois, firent s'approcher d'autres hommes et, le leur indiquant par des signes, ils leur firent comprendre quelle pouvait en être l'utilité. Les hommes qui, ainsi rassemblés, émettaient des sons formés par leur souffle, fixèrent des mots, tels qu'ils les avaient produits dans leur pratique quotidienne, et il advint ensuite, comme conséquence, qu'en désignant de manière répétée les choses d'usage courant, ils commencèrent à parler et créèrent ainsi un langage commun.

2 La découverte du feu ayant donc été à l'origine des premiers groupements humains, des relations entre individus et d'une vie commune, et les hommes qui se rassemblaient en un même lieu ayant, par rapport aux autres êtres animés, le privilège de se mouvoir non pas penchés vers le sol, mais debout, les yeux tournés vers les astres, le privilège aussi d'utiliser leurs mains et leurs doigts pour réaliser facilement toutes sortes de choses, certains des hommes ainsi réunis entreprirent de faire des abris avec des feuillages, d'autres de creuser des grottes au creux des montagnes, quelques-uns, imitant la manière dont les hirondelles bâtissent leur nid, de se faire un refuge avec de la boue et des branchages. Puis observant les abris des autres et apportant du nouveau à ce qu'ils avaient eux-mêmes imaginé, ils amélioraient de jour en jour leurs types de cabanes. 3 Comme ces hommes étaient portés, par nature, à imiter et à s'instruire, fiers de ce qu'ils avaient trouvé, ils se montraient chaque jour les uns aux autres ce qu'ils avaient réussi à bâtir et, stimulant par l'émulation leur ingéniosité, ils devenaient de jour en jour plus avisés. Ils commencèrent par dresser des pieux fourchus, y entrelacèrent des branchages et recouvrirent de boue ces parois. D’autres maçonnèrent des murs avec des blocs de boue séchée et un chaînage de bois, et ils les recouvraient de roseaux et de feuillages pour se protéger des pluies et des fortes chaleurs. Ces couvertures n'ayant pas résisté aux pluies, dans les intempéries hivernales, ils les firent à deux pans et enduisirent de boue les deux versants de la toiture, canalisant ainsi la descente des eaux.

4 Que ces réalisations aient connu les origines décrites ci-dessus, il nous est donné de le constater dans le fait que des nations étrangères utilisent, de nos jours encore, ce type de construction : en Gaule, par exemple, en Espagne, en Lusitanie, en Aquitaine avec des bardeaux de chêne rouvre ou du chaume. En Colchide, dans le Pont, où les forêts sont abondantes, on dispose à droite et à gauche des arbres d'une seule pièce, on laisse entre eux un espace proportionné à leur longueur et l'on dispose transversalement sur ces arbres, à leurs extrémités, d'autres arbres qui circonscrivent l'espace intérieur de l'habitation. De plus, liant ensuite les angles, sur les quatre faces, avec des poutres disposées en alternance, 
et dressant ainsi les murs avec des arbres placés à l'aplomb de ceux du bas, ils élèvent des tours et ils bouchent, à l'aide de copeaux et de boue, les vides que laisse le bois mal dégrossi. Ils mettent par ailleurs en place une toiture, en diminuant à leurs extrémités et avec un resserrement progressif, la longueur des traverses et, à partir des quatre faces jusqu'au point supérieur médian, ils forment ainsi un toit en pavillon qu'ils recouvrent de feuillage et de boue, réalisant un type barbare de couverture « testudinée » (à quatre pans) de tours.

5 Les Phrygiens cependant qui habitent les régions de plaine pauvre en forêts et qui ne disposent donc pas de bois choisissent des buttes naturelles, ils y ouvrent des excavations en leur milieu, percent des passages et élargissent ces espaces autant que le permet la nature du lieu. Liant, par-dessus, de grosses branches les unes aux autres, ils forment un cône qu'ils recouvrent de roseaux et de sarments, et ils entassent d'énormes mottes de terre sur ces habitations. Ce type de couverture assure ainsi des hivers très chauds et des étés très frais. Certains utilisent les plantes des marais comme couverture des huttes qu'ils construisent. Chez d'autres peuples encore et en divers lieux, c'est d'une manière exactement semblable qu'est réalisée la construction des cabanes. Aussi bien nous pouvons voir à Marseille des toitures sans tuiles, faites de paille mélangée à de la terre. À Athènes, il y a, de nos jours encore, sur l'Aréopage, les vestiges de ce passé : une toiture faite de boue. La cabane de Romulus, sur le Capitole ainsi que les toits de chaume des sanctuaires sur la citadelle peuvent donner un témoignage significatif de ces pratiques anciennes.

$6 \mathrm{La}$ conclusion que nous pouvons donc tirer de ces exemples est que tels furent bien, dans les temps anciens, les modes d'invention de l'art de bâtir.

En développant chaque jour davantage leur habileté manuelle comme constructeurs et en stitulant leur ingéniosité d'esprit, ces constructeurs acquirent à l'usage la maîtrise de leur art. Comme il s'ajoutait à cela qu'ils mettaient aussi beaucoup de cœur à l'ouvrage, le résultat fut que ceux qui étaient les plus actifs dans ces travaux purent se présenter comme des hommes du métier. Telle ayant donc été cette histoire originelle et la nature, non seulement ayant doté la race humaine de sens, comme les autres êtres animés, mais lui ayant donné aussi les armes de la pensée et de la réflexion, et ayant soumis à son pouvoir les autres êtres animés, les hommes s'élevèrent, par étapes, de la construction d'habitation jusqu'aux autres arts et sciences, assurant le passage d'une vie sauvage et rude à l'état de civilisation.

Le récit de Vitruve associe l'apparition de la première technique - le feu -, et la socialisation élémentaire - le langage des signes, puis la parole -. Autour de flammes allumées par accident, les hommes se réunissent, puis entreprennent de communiquer et, de la sorte, se constituent en communauté autour d'un foyer. Là, exploitant les ressources de leurs mains, ils édifient des cabanes dont le modèle est emprunté au règne animal. On notera que, pour justifier le lien entre le feu et la construction des abris, il est nécessaire de faire intervenir une discrète analogie religieuse, le rituel de fondation d'une cité, dans lequel le foyer public précède et conditionne l'établissement des foyers privés. Le premier attroupement des sauvages emprunte la forme d'une proto-cité. 
L'analogie donne, significativement, une couleur politique à un épisode des âges primitifs, comme s'il était impossible de concevoir une communauté humaine sans référence à la ciuitas.

À ce stade, le récit des origines bascule dans la description ethnographique, lorsque Vitruve invoque, à l'appui de la reconstitution qu'il propose de la sociogenèse, des exemples tirés des façons de bâtir attestées, de son temps, en Gaule, en Espagne, en Lusitanie, en Aquitaine et en Colchide, en Phrygie, à Marseille et même à Athènes. Suivant ce crescendo, l'espace romain, étendu à l'œcumène, constitue une galerie des techniques, un conservatoire des solutions architecturales dont l'invention a jalonné le passage des premiers abris précaires aux maisons en dur et au toits de tuiles. Ici, les pièces figurant à l'inventaire ethnographique traduisent directement, voire prouvent une chronologie de l'évolution technique, laquelle est la forme sensible du processus de civilisation. Le panorama de l'œcumène alimente une histoire de la culture. Du même coup, parce que, dans l'espace de l'imperium Romanum, coexistent des époques techniques différentes, des cahutes les plus primitives au dernier cri des hommes de l'art, la géographie de l'acculturation aux formes de bâti romaines dessine dans l'espace le cours des âges ; là où les formes se rapprochent le plus du modèle romain, c'est que l'œuvre de la civilisation s'est accomplie. La périégèse du monde romain est un voyage dans le temps. Au terme de sa démonstration, qui n'est pas un récit de la préhistoire humaine, mais une origo de l'architecture, c'est-à-dire un éloge de son art, la civilisation tout entière surgit de l'agencement de l'espace. L'inventaire des techniques de bâti atteste non seulement de l'antique savoir-faire, mais aussi de la compétence supérieure de l'architecte romain, capable d'embrasser et d'exposer tous les savoirs de son art, même étrangers, et qui s'impose ainsi comme un propagateur de cultus, un agent efficace au service du projet impérial romain.

Pierre Cordier

Université de Toulouse II-Le Mirail

UFR d'Histoire, Arts et Archéologie

5, allées Antonio Machado

31058 Tulouse Cedex 9

pierre.cordier@univ-tlse2.fr 